\title{
Aranhas epígeas de um fragmento de mata em área urbana em Fortaleza, Ceará, Brasil
}

\author{
Raul Azevedo ${ }^{1}$, Kamilla Gonçalves Menezes ${ }^{2}$, Raissa Aguiar Barbosa ${ }^{2}$, Joaquin \\ Deusdedit Rocha Matos Neto ${ }^{2}$, José Onofre Nascimento Monteiro ${ }^{2}$, Alysson \\ Guedes Coutinho \& Luis Gonzaga Sales Júnior ${ }^{2}$
}

(1) Universidade Federal do Cariri - Campus Crato, Centro de Ciências Agrárias e da Biodiversidade, Laboratório de Entomologia, Rua Ícaro de Sousa Moreira, Barro Branco, Crato 63130-025, Ceará, Brasil. E-mail: raulbiologo@gmail.com

(2) Universidade Estadual do Ceará, Laboratório de Ornitologia e Sistemática Animal - LORNISA, Avenida Parajana 1700, Itaperi, Fortaleza 60740-000, Ceará, Brasil. E-mail: guedescoutinho@gmail.com

Azevedo R., Menezes K.G., Barbosa R.A., Neto J.D.R.M., Monteiro J.O.N., Coutinho A.G. \& Júnior L.G.S. (2017) Aranhas epígeas de um fragmento de mata em área urbana em Fortaleza, Ceará, Brasil. Pesquisa e Ensino em Ciências Exatas e da Natureza, 1(2): 88-98.

Resumo: A adaptação das aranhas aos diversos tipos de ambientes e suas relações com as comunidades vegetais existentes, as colocam como peças-chaves nos ecossistemas florestais e excelentes organismos bioindicadores devido à sensibilidade aos efeitos da urbanização e fragmentação de ecossistemas. Visando inventariar e investigar a riqueza da fauna de aranhas de solo de um fragmento de mata antropizado, utilizou-se 50 "pitfalls trap" (número total de amostras =600), durante 12 meses no período entre 20102011. Um total de 1238 indivíduos foi coletado, os quais estiveram distribuídos em 51 espécies pertencentes a 18 famílias, das quais Zodariidae e Lycosidae foram as mais abundantes, e Salticidae e Theridiidae as com maior riqueza. Observou-se ainda um alto percentual de espécies raras ("Singletons e Doubletons"). A tendência de não estabilização dos estimadores de riqueza e a relação da predição de espécies coletadas e esperadas indicam que a área precisa de uma melhor amostragem, visando melhor conhecimento da biodiversidade existente e contribuindo para o desenvolvimento para a preservação e conservação da biodiversidade local.

Palavras chave: Araneofauna, aracnídeos, ecossistemas, urbanização.

Epigeal spiders in a forest fragment in an urban area of the city of Fortaleza, Ceará, Brazil

Abstract: Spiders are indicators of environment quality due fact of been sensible to environment changes. Their adaptions among the environment and their relations between vegetal communities make them have a special role in forest ecosystems. However, this relation makes spiders susceptible to effects of urban process and to defloration and ecosystem fragmentation process. A spider fauna survey was developed using 50 "pitfall traps", annual sample process begun in 2010 and finished in 2011 (total samples $=600$ ) in an urbanized patch. A total of 1238 individuals were collected, constituting 51 species belonged to 18 families which Zodariidae and Lycosidae were most abundant, and Salticidae and Theridiidae were the richest families. It's also observed many rare species (Singletons and Doubletons). The richness estimators didn't exhibit a trend do exhibit an asymptote and the relation between collected species and estimated species, suggesting more samples process also suggests a necessity of develop politics to preserve the local biodiversity.

Key words: Araneofauna, arachnids, ecosystems, urbanization.

\section{Introdução}

Aranhas constituem um grupo com riqueza de espécies e abundância relativa elevada, com excelente taxa de adaptação aos diversos tipos de ambientes e ecossistemas existentes 
(Santos et al. 2007). Com 46.587 espécies (World Spider Catalog 2017), as aranhas exercem o controle biológico em comunidades de insetos e outros artrópodes no ambiente natural e em ecossistemas florestais, bem como a regulação dos níveis tróficos de energia (Coddington \& Levi 1991; Wise 1993; Foelix 1996), de modo que os diferentes tipos de plantas combinados com estrutura de vegetação rasteira e serapilheira mais as variáveis climáticas como temperatura e pluviosidade influenciam a composição e abundância de aranhas que habitam o solo (Souza 2007).

A vegetação local atua diretamente na modificação do clima de uma determinada área. Em macroescala, a vegetação cria microclimas e habitats que possibilitam a existência de diversas comunidades de artrópodes (Lawton 1983). Áreas de fragmentos tendem a ter uma redução na diversidade de espécies existentes quando comparadas a áreas maiores, e também áreas com vegetação homogênea tendem a possuir menor complexidade vegetacional, assim como menor quantidade de refúgios (Collinge 1996). Tais tendências também foram observadas por Indicatii et al. (2005) ao amostrar aranhas de duas áreas de fragmento com diferentes graus de ação antrópica. Contudo, áreas de vegetação urbana tendem a ter elevada riqueza de espécies devido a sua complexidade estrutural (Niemelã 1999). Essa tendência em que fragmentos urbanos apresentam maior riqueza de espécies foi observada para ácaros (Daud \& Feres 2005) e em formigas por Morini et al. (2007) ao amostrar formigas de três fragmentos de Mata Atlântica em área antropizada e constatar que a riqueza amostrada foi $21 \%$ maior do que outras áreas. Diversos trabalhos têm mostrado que a diversidade vegetal, estrutural, a arquitetura dos ramos e galhos, e a formação da serapilheira influencia na riqueza de espécies e indivíduos em comunidades de aranhas, devido ao fato de que esses fatores fornecem proteção e forrageamento (Souza \& Martins 2005; Corcuera et al. 2008; Cabra-García et al. 2010).

Contudo, essa diversidade de microclimas e habitats sofre alterações antrópicas que resultam em modificações dos processos de competição, predação e parasitismo em artrópodes (Saunders et al. 1991), causando modificações na dinâmica das comunidades existentes, bem como na fragmentação de ecossistemas urbanos. Os efeitos da fragmentação de ecossistemas e habitats são frequentemente delimitados (e.g., Bolger et al. 2000; Gibb \& Hochuli 2002), e no contexto nacional, quando se trata dos efeitos da fragmentação de ecossistemas florestais urbanos em comunidades de aranhas, percebe-se a carência de dados sobre tal fato (Dias et al. 2006).

O presente trabalho teve o objetivo de realizar um inventário da fauna de aranhas existentes em um fragmento de Tabuleiro Litorâneo no interior da Universidade Estadual do Ceará, nordeste do Brasil.

\section{Material e Métodos}

\section{Área de pesquisa}

O presente trabalho foi realizado em um fragmento de Tabuleiro Litorâneo (7.76 hectares) no interior da Universidade Estadual do Ceará, Campus do Itaperi, Fortaleza, Ceará (Figura 1) (0347'42.08" S, 38³3'32.89" W), nordeste do Brasil.

O Campus Itaperi apresenta poucos elementos oriundos da sua vegetação original (Tabuleiro Litorâneo), restando apenas pequenas faixas e testemunhos de mata que foram substituídos progressivamente por ambientes urbanos, agro-pastorís, fragmentos de mata secundária (Tabuleiro Pré-Litorâneo) (Figura 2), ambiente lacustre e ribeirinho (Sales Júnior 1999). A área onde a pesquisa foi realizada consiste em um fragmento de mata secundária, a qual a vegetação apresenta porte arbóreo e arbustivo, com algumas árvores frutíferas.

O Tabuleiro litorâneo é uma vegetação caracterizada por árvores cuja altura varia entre 3 e 5 metros, exibindo em alguns casos a presença de espécies do Cerrado e Cerradão (Fernandes 1998), constituindo assim florestas estacionais semi-decíduas, distintas da Caatinga (Castro et al. 2012; Moro et al. 2015). 


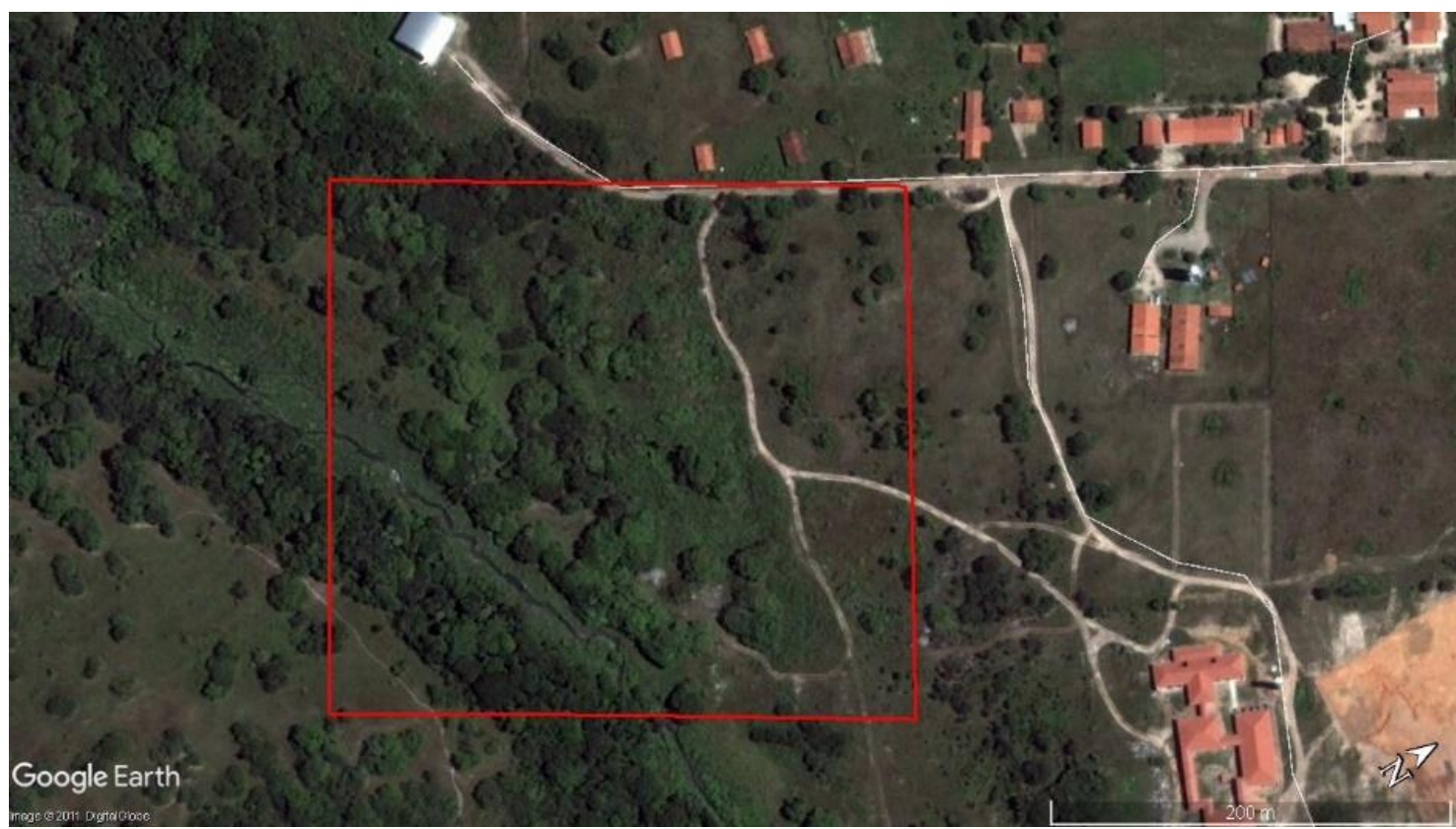

Figura 1. Foto de Satélite do Fragmento de Tabuleiro Pré-Litorâneo (área dentro do quadrado) existente no interior da Universidade Estadual do Ceará, Campus Itaperi.

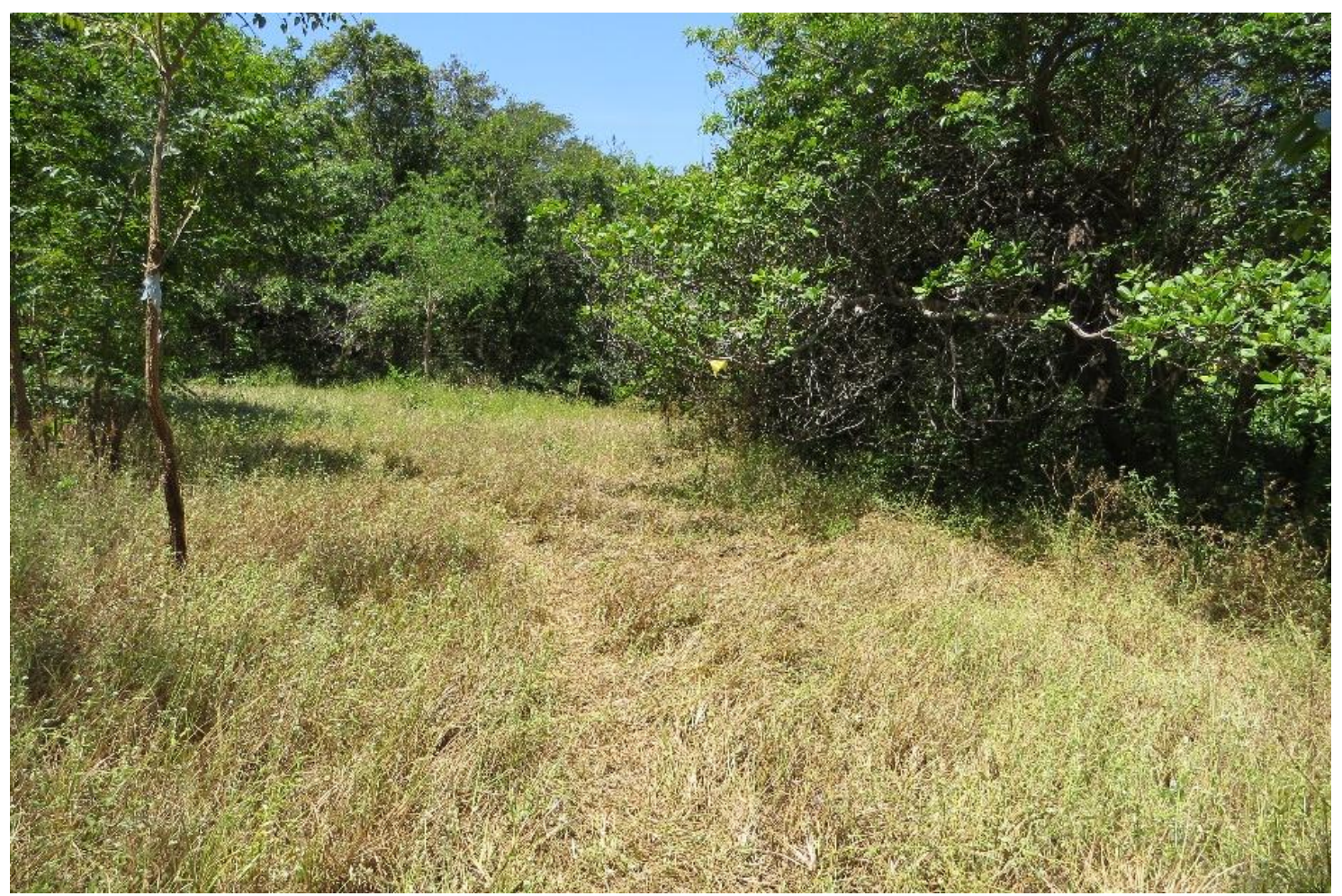

Figura 2. Fragmento de Tabuleiro Litorâneo onde foi realizada a pesquisa.

\section{Amostragem e obtenção dos dados}

A amostragem das aranhas foi conduzida por meio de armadilhas do tipo "pitfall trap" (15 cm de profundidade, $10 \mathrm{~cm}$ de diâmetro, sem o uso de telhado de isopor), com início em agosto de 2010 e término em julho de 2011, totalizando 12 coletas mensais. Em cada amostragem foram instaladas 50 armadilhas, espaçadas entre si a $10 \mathrm{~m}$, dispostas em cinco transectos, as quais permaneceram operantes por sete dias (Carvalho 2015). Em cada armadilha foi utilizada uma solução conservante de álcool à $70 \%$, com monoetileno glicol à $30 \%$, com 
algumas gotas de detergente para quebrar a tensão superficial do líquido e cada armadilha foi considerada uma unidade amostral.

Dados meteorológicos foram obtidos por meio da Fundação Cearense de Meteorologia FUNCEME - Funceme (2011).

O material coletado foi identificado pelo taxonomista Dr. Antônio Domingos Brescovit (Seção de Artrópodes Peçonhentos, Instituto Butantan, Universidade de São Paulo) e encontra-se tombado na Coleção de Arachnida e Myriapoda do Instituto Butantan, São Paulo, Brasil (IBSP curador A.D. Brescovit).

\section{Análises dos dados}

Cada armadilha foi considerada uma unidade amostral, representando assim 50 unidades amostrais por coleta. Apenas os indivíduos adultos foram incluídos nas análises. Foram calculados modelos de curva de acumulação de espécies baseados na acumulação de indivíduos e os estimadores de riqueza: Chao I, ChaoII, Jackknife I e Jackknife II como forma de predizer o número de espécies existentes na área e mensurar o esforço amostral empregado (Colwell \& Conddington 1994; Chazdon et al. 1998), baseando-se nos parâmetros "uniques” e "duplicates", respectivamente, para Jackknife I e Jackknife II, e os parâmetros "singletons” e "doubletons", respectivamente, Chao I e Chao II (Dias 2004a). Os estimadores de riqueza foram estimados por meio do programa EstimateS versão. 9.1.0 (Colwell 2013), utilizando-se 100 aleatorizações, sem reposição de amostras.

Para análise da variação da diversidade anual, foram calculados os Índices de Shannon e Simpson por meio do programa PAST, versão 3.14.3 (Hammer et al. 2001). Os índices de diversidade são baseados na abundância proporcional das espécies, podendo ser utilizados em monitoramentos ambientais assumindo que alterações adversas no ambiente refletirão em uma redução da diversidade (Magurran 1988).

Variações na composição da fauna e da diversidade ao longo do ano foram mensuradas, respectivamente, por meio do teste não-paramétrico de Kruskal-Wallis e por teste t (Zar 1984), realizados pelo programa PAST, versão 3.14.3. Os efeitos da pluviosidade na riqueza de espécies e abundância dos indivíduos foram mensurados por meio de um GLM's ("Generalized linear models”), usando distribuição gaussiana, por meio do programa PAST, versão 3.14.3. Optou-se por utilizar GLM por este assumir o uso de distribuições não normais e por assumir independência das observações (Legendre et al. 2002).

\section{Resultados}

Foram coletados 1305 indivíduos, dos quais 1238 são indivíduos adultos, pertencentes a 18 famílias, classificados em 51 espécies (Tabela 1). Os 67 indivíduos juvenis foram classificados nas seguintes famílias: Linyphiidae (quatro indivíduos); Lycosidae (41 indivíduos de Trochosa); Oxyopidae (seis indivíduos); Pholcidae (um indivíduo de Mesabolivar); Salticidae (10 indivíduos); Theraphosidae (um indivíduo de Acanthoscurria) e Zodariidae (quatro indivíduos de Leprolochus). Nenhuma família foi representada apenas por indivíduos juvenis.

Os morfotipos com maior número de indivíduos coletados foram Leprolochus sp. 1 (Zodariidae) e Trochosa sp. 1 (Lycosidae). As famílias com maior riqueza de espécies foram Salticidae (16) e Theridiidae (cinco). Ao todo, 17 espécies foram consideradas "singletons" e cinco espécies foram consideradas “doubletons”.

Fevereiro foi o mês com maior número de espécies amostrado $(\mathrm{N}=30)$, seguido por julho e agosto, com 20 e 19, respectivamente (Tabela 1) e o menor número foi em dezembro, com apenas quatro espécies coletadas. Em termos de abundância de indivíduos, os meses com maior número foram janeiro e fevereiro, com 167 e 166 indivíduos, respectivamente (Figura 3). A composição da fauna variou significativamente ao longo do ano (Teste de Kruskal-Wallis, $\mathrm{U}=$ 37.92, $\mathrm{p}<0.05$ ). Os modelos GLM's plotados para as relações entre pluviosidade e abundância, e pluviosidade e riqueza de espécies exibiram relações positivas, respectivamente $(\mathrm{y}=0.38347 \mathrm{x}+$ $60.327)(\mathrm{p}<0.05)$ e $(\mathrm{y}=4.9067 \mathrm{x}+30.383)(\mathrm{p}<0.05)$. 
Ao comparar a curva de acumulação de espécies por meio do número de espécies observadas ( $\mathrm{S}$ obs $=51$ ), com os estimadores de riqueza (Figura 4), percebe-se que o estimador Chao I exibiu tendência a estabilizar, enquanto que os outros estimadores não exibiram tendência a estabilização. Os estimadores variaram suas estimativas de riqueza de espécies entre 68 espécies (Jackknife I) e 78.4 espécies (Jackknife II).

O índice de Simpson (Figura 5) não exibiu variação significativa entre os meses analisados. Contudo, o índice de Shannon exibiu variação significativa entre os meses analisados.

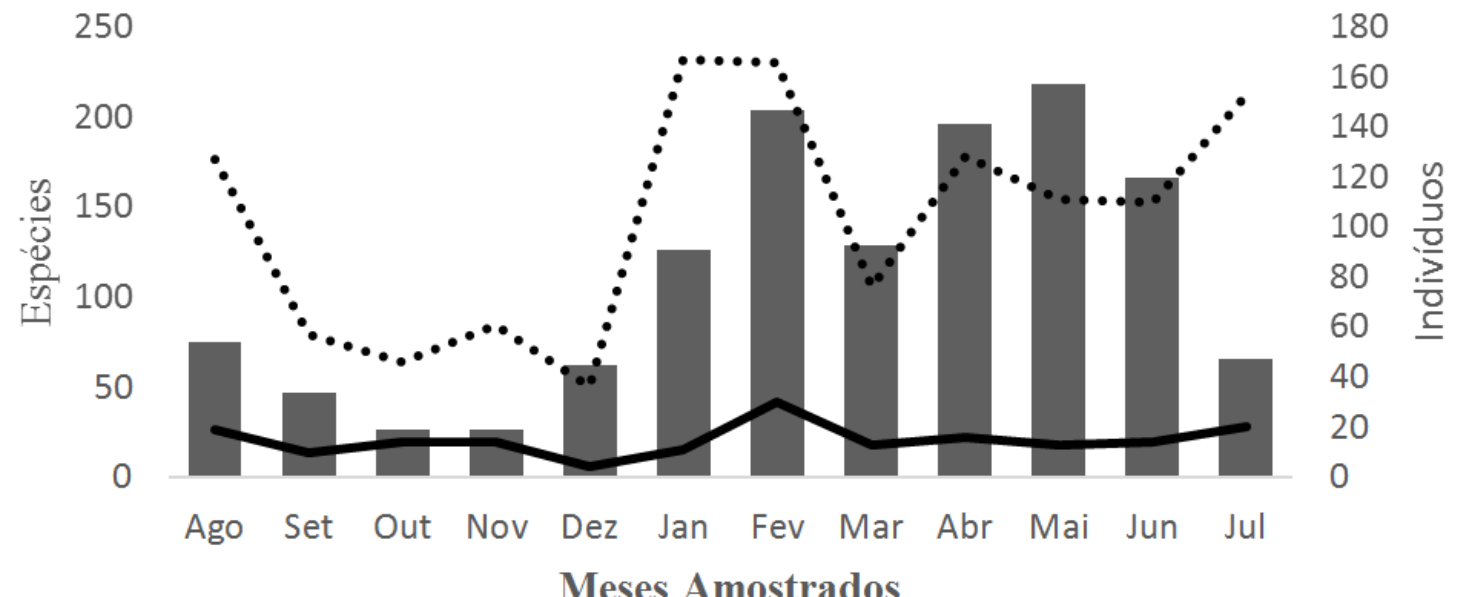

$$
\text { Pluviosidade } \quad \text {...... Abundância Espécies Obs }
$$

Figura 3. Variação anual da riqueza e abundância em função da pluviosidade para o período de agosto de 2010 a junho de 2011.

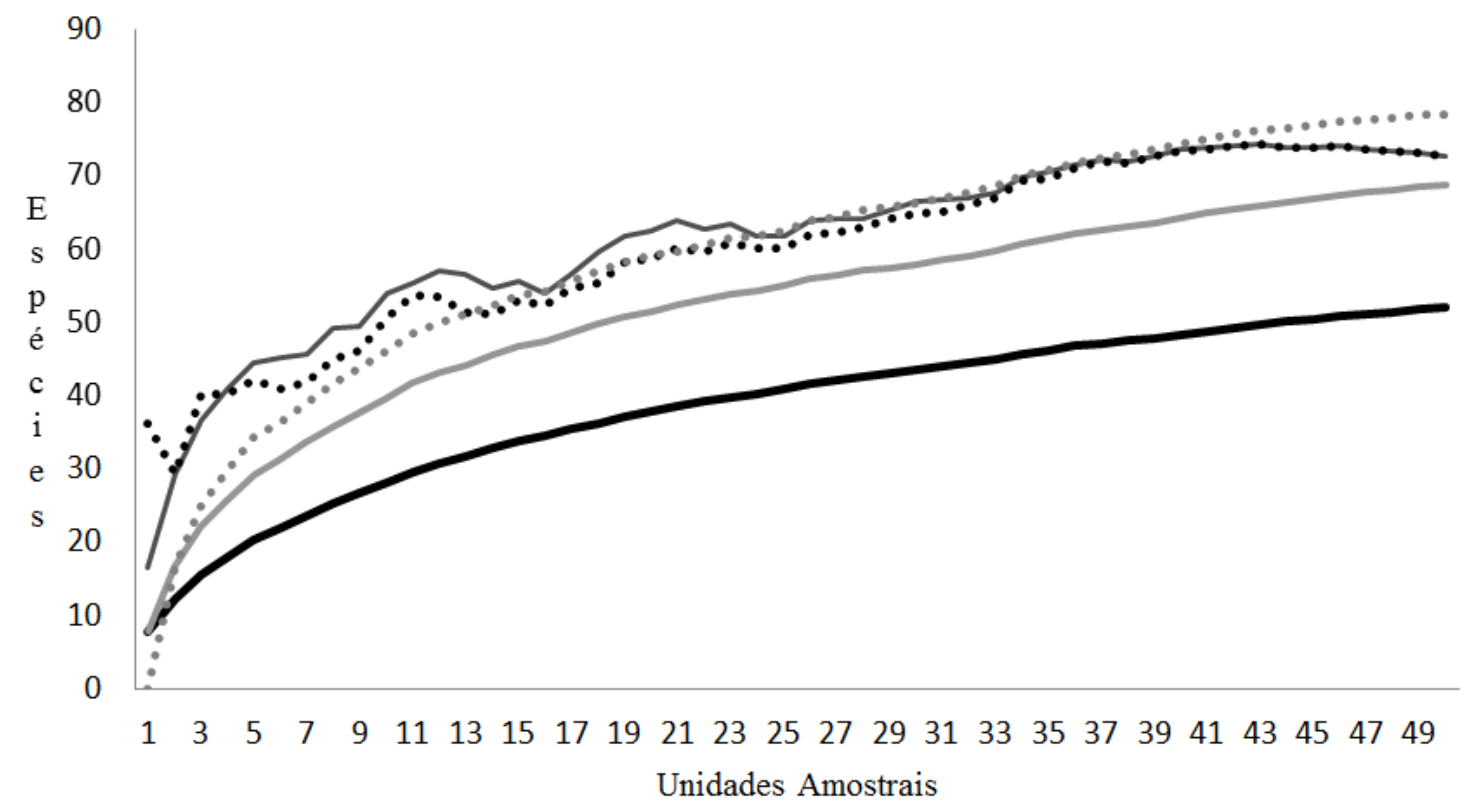

$\longrightarrow$ SOBS $\longrightarrow$ Chao I ...... Chao II $\longrightarrow$ Jack-Knife I .... Jack-Knife II

Figura 4. Estimadores de riqueza para área de pesquisa para o período de agosto de 2010 a julho de 2011. Legenda: S OBS: Espécies Observadas; Chao I: Estimador Chao de primeira ordem; Chao II: Estimador de segunda ordem; Jack-Knife I: Estimador Jack-Knife de primeira ordem; Jack-Knife II: Estimador de Riqueza de segunda ordem. 
Tabela 1. Composição da fauna de aranhas coletada durante o período de agosto de 2010 a julho de 2011, total de indivíduos coletados por mês (50 unidades amostrais) e pluviosidade observada no Campus da Universidade Estadual do Ceará.

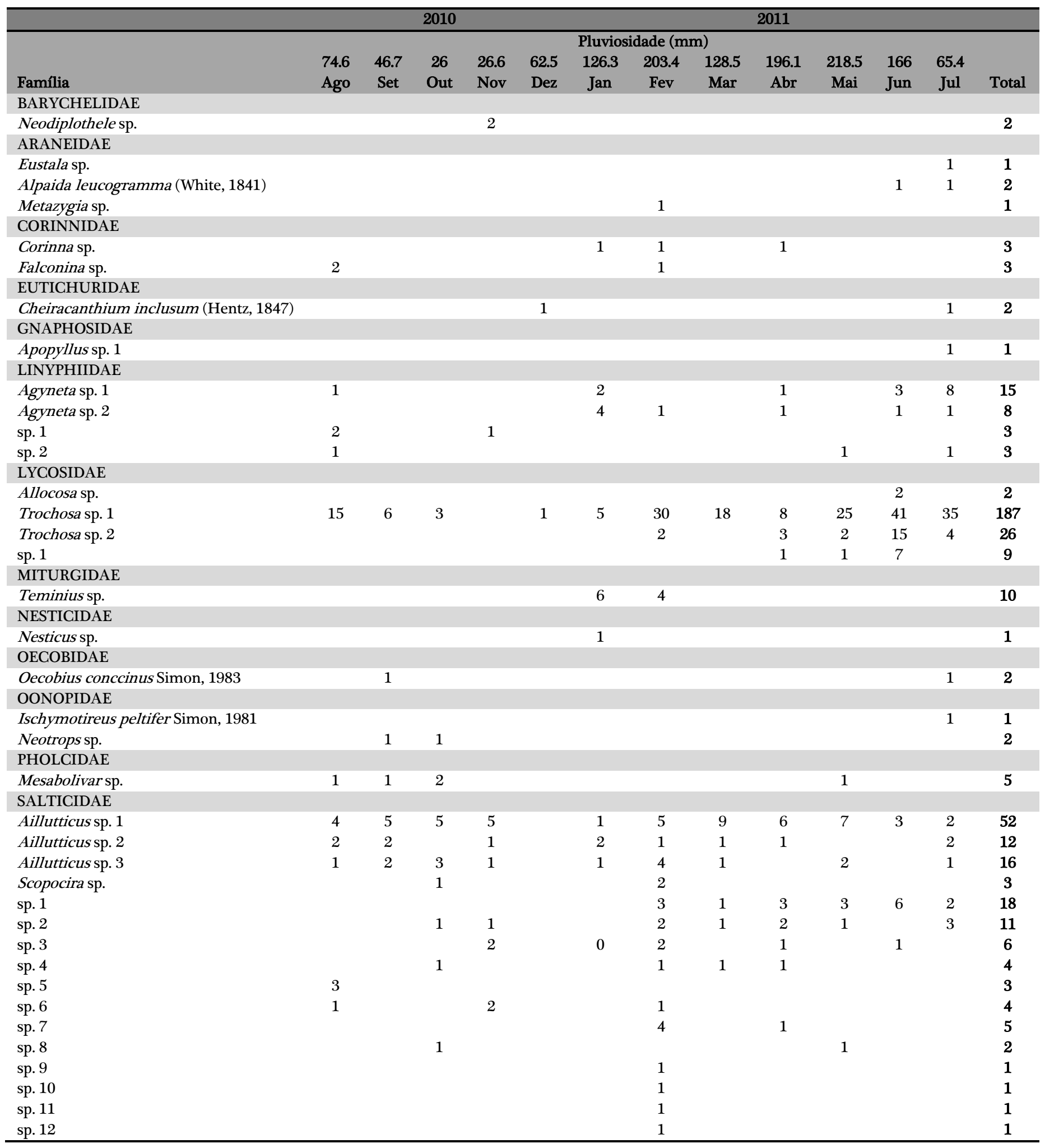


Tabela 2. Composição da fauna de aranhas coletada durante o período de agosto de 2010 a julho de 2011, total de indivíduos coletados por mês (50 unidades amostrais) e pluviosidade observada no Campus da Universidade Estadual do Ceará (continuação).

\begin{tabular}{|c|c|c|c|c|c|c|c|c|c|c|c|c|c|}
\hline \multirow[b]{4}{*}{ Família } & \multicolumn{4}{|c|}{2010} & \multicolumn{9}{|c|}{2011} \\
\hline & \multicolumn{12}{|c|}{ Pluviosidade (mm) } & \multirow[b]{3}{*}{ Total } \\
\hline & 74.6 & 46.7 & 26 & 26.6 & 62.5 & 126.3 & 203.4 & 128.5 & 196.1 & 218.5 & 166 & 65.4 & \\
\hline & Ago & Set & Out & Nov & Dez & Jan & Fev & Mar & Abr & Mai & Jun & Jul & \\
\hline \multicolumn{14}{|l|}{ TETRAGNATHIDAE } \\
\hline Glenognatha sp. & & & & & & & & & & & 1 & 2 & 3 \\
\hline Leucage sp. & & & & & & & & & & & 1 & & 1 \\
\hline \multicolumn{14}{|l|}{ THERAPHOSIDAE } \\
\hline Magulla sp. & 1 & & & & & & & & & & & & 1 \\
\hline Acanthoscurria sp. & & & & & & & 1 & & & & & & 1 \\
\hline \multicolumn{14}{|l|}{ THERIDIIDAE } \\
\hline Coleosoma floridanum Banks, 1900 & & & & & & & & & & & & 2 & 2 \\
\hline Euryopis sp. & 6 & & & 1 & & 1 & 1 & 6 & & & & & 15 \\
\hline Steatoda sp. 1 & 10 & 2 & 1 & 1 & & 6 & 12 & & & & & 3 & 35 \\
\hline Steatoda sp. 2 & & & & & 1 & & & & & & 1 & & 2 \\
\hline \multicolumn{14}{|l|}{ THOMISIDAE } \\
\hline Misumenops sp. & & & & & & & & 1 & & & & & 1 \\
\hline sp. 1 & & & & 1 & & & & & & & & & 1 \\
\hline \multicolumn{14}{|l|}{ ZODARIIDAE } \\
\hline Cybaedamus sp. & & & & & & & 9 & 1 & & 1 & & & 11 \\
\hline Leprolochus sp. 1 & 55 & 17 & 6 & 27 & 31 & 135 & 51 & 31 & 87 & 65 & 25 & 81 & 611 \\
\hline \multirow[t]{2}{*}{ Leprolochus sp. 2} & 13 & 16 & 16 & 13 & 3 & & 9 & 1 & 6 & & & 2 & 79 \\
\hline & 118 & 53 & 41 & 58 & 37 & 165 & 161 & 73 & 123 & 111 & 108 & 156 & \\
\hline
\end{tabular}

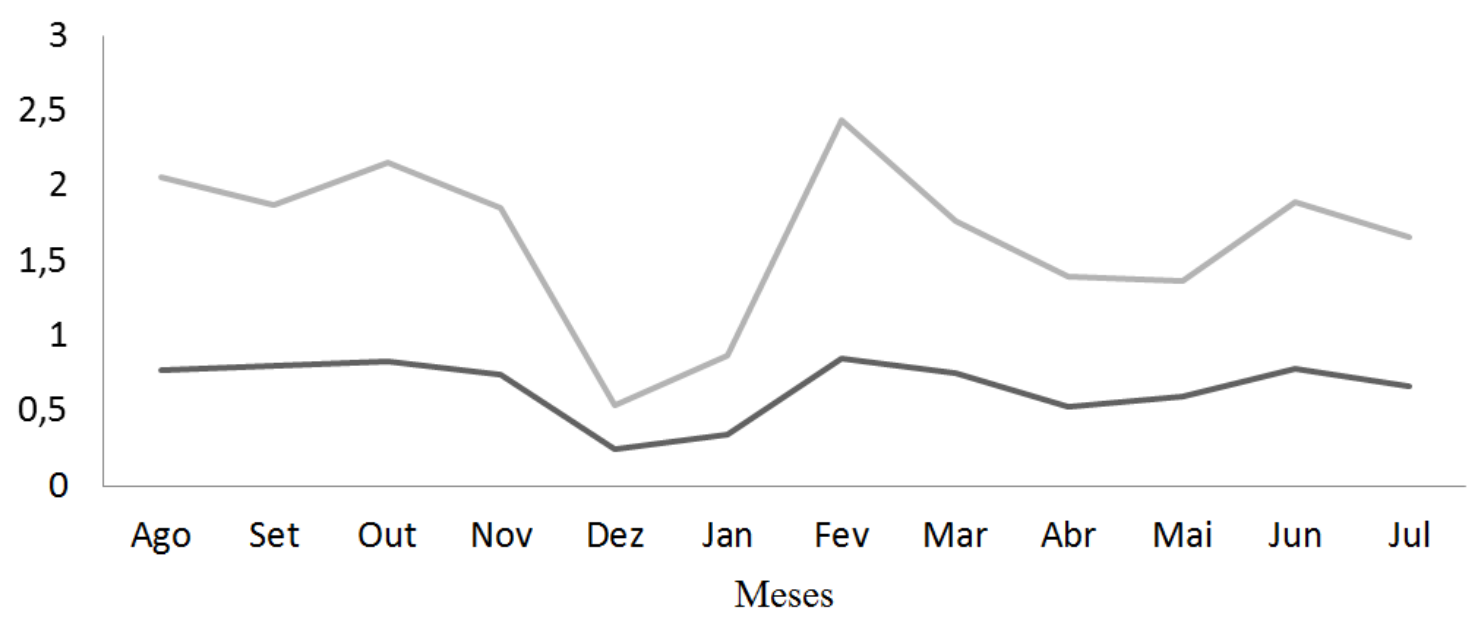

—Índice de Simpson —Índice de Shannon

Figura 5. Variação da Diversidade ao longo do período de agosto de 2010 a julho de 2011.

\section{Discussão}

A riqueza de espécies de assembleias de artrópodes urbanos é correlacionada com dois parâmetros: a heterogeneidade do ambiente e sua composição (Sattler et al. 2010). As assembleias de aranhas são influenciadas pela heterogeneidade ambiental e por mudanças antropogênicas, tais como a urbanização e a fragmentação de habitats e ecossistemas (Shochat et al. 2004; Melo et al. 2011), tais mudanças influenciam diretamente as dinâmicas existentes dentro das comunidades, tais como número de espécies, indivíduos e sua composição (Whitmore et al. 2002), levando-as ao decréscimo ou extinção (Magura et al. 2010). 
Em inventários de aranhas de solo realizados em fragmentos em áreas urbanas ou antropizadas, as famílias mais abundantes costumam ser: Linyphiidae (Candiani et al. 2005; Indicatii et al. 2005), Salticidae (Dias 2004b; Dias et al. 2005); Lycosidae e Zodariidae (Cunha et al. 2012).

Diferente de Candiani et al. (2005) e Indicatii et al. (2005), cujos inventários de aranhas de solo em fragmentos reportam a família Linyphiidae como mais abundante, as famílias Zodariidae e Lycosidae foram consideradas as mais abundantes em nosso estudo (respectivamente, $\mathrm{N}=690$ e $\mathrm{N}=232$ ), similarmente ao trabalho de Cunha et al. (2012) em áreas de Cerrado com diferentes graus de fragmentação e ação antrópica. Tais diferenças na abundância e composição das famílias nos estudos podem ser atribuídas ao tipo de vegetação existente e, consequentemente, na formação da serapilheira, estando diretamente relacionada a sítios para exploração de refúgios, locais para postura de ovos, quantidade de presas (Rypstra et al. 1999) e aos diferentes estágios de sucessão existentes (Willett 2001).

Muitos representantes da família Zodariidae são bem adaptados a ambientes secos ou com pouca humidade no solo, exibindo alta riqueza de espécies e abundância de indivíduos (Jocqué 1991). Leprolochus é um gênero de ocorrência sul americano em ambientes cuja precipitação varia de média a alta, encontrados em áreas não florestais ou de vegetação arbustiva (Jocqué 1988). O alto número de indivíduos de Leprolochus sp. pode ser visto como indicativo de área degrada, concordando com Gibb \& Hochuli (2002), onde a maior parte das diferenças encontradas entre fragmentos de áreas naturais e áreas não fragmentadas em um ecossistema australiano, estava relacionado aos indivíduos da família Zodariidae.

A grande quantidade de indivíduos de Lycosidae coletados pode ser atribuída ao processo de fragmentação existente da vegetação, que transformou uma área florestal em área aberta, propiciando a existência de licosídeos (Topping \& Sunderland 1992; Capocasale 2001; Jocqué \& Alderweireldt 2005). Inventários realizados em fragmentos florestais em diversas localidades não reportam a ocorrência de licosídeos (Candiani et al. 2005; Dias et al. 2006; Bonaldo \& Dias 2010), sugerindo assim a sua preferência por áreas abertas.

A baixa abundância de Araneidae e Tetragnathidae se deve ao fato dessas aranhas normalmente habitarem o extrato médio das florestas (Candiani et al. 2005). Do mesmo modo, a baixa abundância também pode ser atribuída ao método utilizado ("pitfall trap"), voltado para a coleta de aranhas epígeas, mas também a ausência de vegetação secundária e terciária (Melo et al. 2011).

Salticidae é a família mais abundante em solos de regiões neotropicais devido ao favorecimento das altas temperaturas (Jocqué 1984). A quantidade de espécies coletadas, bem como o número de indivíduos sugere que essas aranhas sejam mais adaptadas a habitar ambientes fragmentados, com vegetação aberta e maiores alterações ambientais, como sugerido por Benati et al. (2005).

A abundância da maioria das espécies foi inferior a cinco indivíduos, justificando assim a variação significativa do Índice de Shannon, e seu pico nos meses de janeiro e fevereiro (Figura 5). A abundância e riqueza encontrada variaram concomitantemente com a pluviosidade, exibindo maiores valores com os maiores picos de pluviosidade (Funceme 2011) para a região (Figura 3). Esses valores são justificados pela sensibilidade a alterações ambientais (Whtimore et al. 2002), e a uma maior quantidade de presas e respectivas fontes alimentares que tendem a ser maiores nas estações chuvosas em ecossistemas florestais tropicais (Cardoso et al. 2007).

A não estabilização da curva de acumulação de espécies se deve ao fato de que muitas espécies foram consideradas "singletons" e "doubletons", resultado corroborado por outros estudos (e.g., Indicatti et al. 2005), contudo, a curva de acumulação é uma aproximação das médias dos estimadores de riqueza utilizados, indicando assim que a amostragem estaria próxima de estar completada (Cabra-García et al. 2010).

Candiani et al. (2005) sugerem em seu artigo que os incêndios ocorridos nas áreas de pesquisa podem ter ajudado a diminuir a riqueza da área, resultando na quantidade de “singletons" e “doubletons”. Contudo, a literatura mostra que para áreas de Cerrado, o fogo não 
afeta o número de espécies de aracnídeos, reduzindo apenas a sua abundância (Freire Jr. \& Motta 2011).

Esperava-se encontrar diferenças mais discrepantes ao se comparar os valores referentes a riqueza de espécies observadas e riqueza estimada, visto que todos os estimadores apresentaram tendência a não estabilização, exceto o estimador Chao I. Uma amostragem com maior esforço amostral, baseada em outras metodologias poderia fazer com que as curvas exibissem tendência a estabilidade, resultando em um maior número de espécies coletadas.

Os resultados obtidos permitem inferir temporalmente e localmente que a composição da fauna de aranhas de solo de fragmentos de áreas de Tabuleiro litorâneo difere de áreas florestais, principalmente quando representam resquícios de Mata Atlântica. A ocorrência de áreas abertas e ou com baixa camada da serapilheira contribuiu para a ocorrência dos indivíduos e elevada abundância de Lycosidae e Zodariidae, e por sua vez, contribuindo para a baixa abundância de Linyphiidae.

O presente trabalho constitui o primeiro inventário de fauna de aranhas do município de Fortaleza. Espera-se que este trabalho possa incentivar novas pesquisas, principalmente nas áreas de preservação existentes no município, e desse modo, contribuindo para o conhecimento da araneofauna local e para a preservação do meio ambiente.

\section{Agradecimentos}

Ao Dr. Antônio D. Brescovit pela identificação dos espécimes coletados. Aos avaliadores anônimos pela revisão crítica do manuscrito.

\section{Referências}

Benati K.R., Souza-Alves J.P., Silva E.A., Peres M.C.L. \& Coutinho E.O. (2005) Aspectos comparativos das comunidades de aranhas (Araneae) em dois remanescentes de Mata Atlântica do Estado da Bahia, Brasil. Biota Neotropica, 5(1A): 79-87.

Bolger D.T., Suarez A.V., Crooks K.R., Morrison S.A. \& Case T.J. (2000) Arthropods in urban fragments in Southern California: area, age and edge effects. Ecological Applications, 10(4): 1230-1248.

Bonaldo A.B. \& Dias S.C. (2010) A structured inventory of spiders (Arachnida, Araneae) in natural and artificial forest gaps at Porto Urucu, Western Brazil, Amazonia. Acta Amazonica, 40(2): 357-372.

Cabra-García J., Chacón P. \& Valderrama-Ardila C. (2010) Additive partitioning of spider diversity in a fragmented tropical dry forest (Valle del Cauca, Colombia). Journal of Arachnology, 38: 192-205.

Candiani D.F., Indicatti R.P. \& Brescovit A.D. (2005) Composição e diversidade da araneofauna (Araneae) de serapilheira em três florestas urbanas na cidade de São Paulo, São Paulo, Brasil. Biota Neotropica, 5(1A): 111-123.

Capocasale R.M. (2001) Redescripción de Lycosa poliostoma (C. L. KOCK) (Araneae, Lycosidae). Revista Iberica de Aracnología, 3: 79-86.

Cardoso P., Silva I., De Oliveira N. \& Serrano A.R.M. (2007) Seasonality of spiders (Araneae) in Mediterranean ecosystems and its implications in the optimum sampling period. Ecological Entomology, 32: 516-526.

Carvalho L.S. (2015) Aracnídeos: quem são, por que estudá-los e como coletá-los? (p. 103-140). In: Lima M.S.C.S., Carvalho L.S. \& Prezoto F. (Orgs). Métodos em Ecologia e Comportamento Animal. Teresina: Edufpi. 132 p.

Castro A.S.F., Moro M.F. \& Menezes M.O.T. (2012) O Complexo Vegetacional da Zona Litorânea no Ceará: Pecém, São Gonçalo do Amarante. Acta Botanica Brasilica, 26(1): 108-124.

Chazdon R.L., Colwell R.K., Denslow J.S. \& Guariguata M.R. (1998) Statistical methods for estimating species richness of woody regeneration in primary and secondary rain forests of Northeastern Costa Rica (p. 285-309). In: Dallmeier F. \& Comiskey J.A. (Eds). Forest 
biodiversity research, monitoring and modeling: conceptual background and old world case studies. Paris: Unesco. 671 p.

Coddington J.A. \& Levi H.W. (1991) Systematics and evolution of spiders (Araneae). Annual Review of Ecology and Systematics, 22: 565-592.

Collinge S.K. (1996) Ecological consequences of habitat fragmentation: implications for landscape architecture and planning. Landascape and Urban Planning, 36: 59-77.

Colwell R.K. (2013) EstimateS: Statistical estimation of species richness and shared species from samples. Version 9.1.0. User's Guide and application. Disponível em: http://purl.oclc.org/estimates. 2013 (Acessado em 10/01/2013).

Colwell R.K. \& Conddington J.A. (1994) Estimating terrestrial biodiversity through extrapolation. Philosophical Transactions of the Royal Society of London Biological Sciences, 345: 101-118

Corcuera P., Jiménez M.L. \& Valverde P.L. (2008) Does the microarchitecture of Mexican dry forest foliage influence spider distribution. Journal of Arachnology, 36: 552-556.

Cunha J.A.S., Cristina A., Castro A.A.J.F. \& Brescovit A.D. (2012) Diversidade preliminar de aranhas de solo em áreas de Cerrado Litorâneo com diferentes níveis de conservação, Maranhão, Brasil. Revista Biociências, 18 (1): 5-13.

Daud R.D. \& Feres R.J.F. (2005) Diversidade e Flutuação Populacional de Ácaros (Acari) em Mabea fistulifera Mart. (Euphorbiaceae) de Dois Fragmentos de Mata Estacional Semidecídua em São José do Rio Preto, SP. Neotropical Entomology, 34(2): 191-201.

Dias S.C. (2004a) Planejando estudos de diversidade e riqueza: uma abordagem para estudantes de graduação. Acta Scientiarum. Biological Sciences, 26 (4): 373-379.

Dias M.F.R. (2004b) Levantamento das aranhas de solo (Arachnida: Araneae) na Reserva Biológica de Una, Bahia, Brasil. Sitientibus, 4(1/2): 3-6.

Dias M.F.R., Brescovit A.D. \& Menezes M. (2005) Aranhas de solo (Arachnida: Araneae) em diferentes fragmentos florestais no sul da Bahia, Brasil. Biota Neotropica, 5(1a): 1-10.

Dias S.C., Brescovit A.D., Couto E.C.G. \& Martins C.F. (2006) Species richness and seasonality of spiders (Arachnida, Araneae) in an urban Atlantic Forest Fragment in Northeast, Brazil. Urban Ecosystystem, 9: 323-335.

Fernandes A. (1998) Fitogeografia Brasileira. Fortaleza: Multigraf. 339 p.

Foelix R.F. (1996) Biology of Spiders. $2^{\circ}$ edition. New York: Oxford University Press. 281 p.

Freire Jr.G.B. \& Motta P.C. (2011) Effects of experimental fire regimes on the abundance and diversity of cursorial arachnids of Brazilian savannah (cerrado biome). The Journal of Arachnology, 39: 263-272.

Funceme (2011) Fundação Cearesense de Metereologia e Recursos Hídricos. Disponível em: http://www.funceme.br/index.php/areas (Acessado em 01/08/2011).

Gibb H. \& Hochuli D.F. (2002) Habitat fragmentation in an urban environment: large and small fragments support different arthropod assemblages. Biological Conservation, 106: 91-100.

Hammer O., Harper D.A.T. \& Ryan P.D. (2001) PAST: Paleontological statistics software package for education and data analyses. Disponível em: http://folk.uio.no/ohammer/past/ (Acessado em 10/05/2016).

Indicatti R.P., Candiani D.F., Brescovit A.D. \& Japyassú H.F. (2005) Diversidade de aranhas (Arachnida, Araneae) de solo na bacia do reservatório do Guarapiranga, São Paulo, São Paulo, Brasil. Biota Neotropica, 5(1A): 151-162.

Jocqué R. (1984) Considérations concernant l' abondance relative des araignées errantes et des araignées à toile vivant au niveau du sol. Revue Arachonologique, 5(4): 193-204.

Jocqué R. (1988) An updating of the genus Leprolochus (Araneae: Zodariidae). Studies on Neotropical Fauna and Environment, 23(2): 77-87.

Jocqué R. (1991) A generic revision of the spider family Zodariidae (Araneae). Bulletin of the American Museum of Natural History, 201: 1-160.

Jocqué R. \& Alderweireldt M. (2005) Lycosidae: the grassland spiders. Acta Zoologica Bulgarica, supplement 1: 125-130. 
Lawton J.H. (1983) Plant architeture and the diversity of phytphagous insects. Annual Review of Entomology, 28: 23-29.

Legendre P., Dale M.R.T., Fortin M.J., Gurevitch J., Hohn M. \& Myers D. (2002) The consequences of spatial structure for the design and analysis of ecological field surveys. Ecography, 25: 601-615.

Magura T., Horváth R. \& Tóthmérész B. (2010) Effects of urbanization on ground dewling spiders in forest patches, in Hungary. Lanscape Ecology, 25: 621-629.

Magurran A.E. (1988) Ecological Diversity and Its Measurement. New Jersey: Princeton University Press. 179 p.

Melo T.S., Andrade A.R.S., Benati K.R., Peres M.C.L. \& Dias M.A. (2011) Panorama da araneofauna de fragmentos florestais em Salvador, Bahia, Brasil. Sitientibus, 11(1): 37-47.

Morini M.S.C., Munhae C.B., Leung R., Candiani D.F. \& Voltolini J.C. (2007) Comunidades de formigas (Hymenoptera, Formicidae) em fragmentos de Mata Atlântica situados em áreas urbanizadas. Iheringia, 97(3): 246-252.

Moro M.F., Macedo M.B., De Moura-Fé M.M., Castro A.S.F. \& Da Costa R.C. (2015) Vegetação, unidades fitoecológicas e diversidade paisagística do estado do Ceará. Rodriguésia, 66(3): 717-743.

Niemelã J. (1999) Ecology and urban planning. Biodiversity and Conservation, 8: 119-131.

Rypstra A.L., Wieg C., Balfour R.A. \& Marshall S.D. (1999) Architectural features of agricultural habitats and their impact on the spiders inhabitants. Journal of Arachnology, 27: 371-377.

Sales Júnior L.G. (1999) Distribuição estratigráfica da ornitofauna do Campus do Itaperi da Universidade Estadual do Ceará, (UECE), Fortaleza, CE. Ciências e Tecnologia, 1: 7-16.

Santos A.J., Brescovit A.D. \& Japyassú H.F. (2007) Diversidade de aranhas: sistemática, ecologia e inventários de fauna (p. 1-23). In: Santos A.J., Gonzaga M.O. \& Japyassú H.F. (Eds). Ecologia e Comportamento de Aranhas. Rio de Janeiro: Editora Interciência. 350 p.

Sattler T., Duelli P., Obrist M.O., Arlettaz R. \& Moretti M. (2010) Response of arthopod species richness and functional groups to urban habitat structure and management. Lanscape Ecology, 25: 941-954.

Saunders D.A., Hobbs R.J. \& Margules C.R. (1991) Biological consequences of ecosystem fragmentation: a review. Conservation Biology, 5: 18-32.

Shochat E., Stefanov W.L., Whitehouse M.E.A. \& Faeth S.H. (2004) Urbanization and spider diversity: influences of human modification of habitat structure and productivity. Ecological Applications, 14(1): 268-280.

Souza A.L.T. (2007) Influência da estrutura do habitat na abundância e diversidade de aranhas (p. 25-44). In: Santos A.J., Gonzaga M.O. \& Japyassú H.F. (Eds). Ecologia e Comportamento de Aranhas. Rio de Janeiro: Editora Interciência. 350 p.

Souza A.L.T. \& Martins R.P. (2005) Foliage density of branches and distribution of plant-dwelling spiders. Biotropica, 37: 416-420.

Topping C.J. \& Sunderland K.D. (1992) Limitations to the use of pitfall traps in ecological studies exemplified by a study of spiders in a field of winter wheat. Journal of Applied Ecology, 29: 485-491.

Whitmore C., Slotow R., Crouch T.E. \& Dippenaar-Schoeman A. (2002) Diversity of spiders (Araneae) in Savanna Reserve, Northern Province, South Africa. Journal of Arachnology, 30: 344-356.

Willett T.R. (2001) Spiders and other arthropods as indicators in old-groth versus logged redwood stands. Restoration Ecology, 9: 410-420.

Wise D.H. (1993) Spiders in Ecological Webs. New York: Cambridge University Press. 328 p.

World Spider Catalog (2017) World Spider Catalog. Natural History Museum Bern. Disponível em: http://wsc.nmbe.ch, version 18.0 (Acessado em 10/04/2017).

Zar J.H. (1984) Biostatistical analysis. $2^{\circ}$ edition. New Jersey: Prentice-Hall. 719 p. 